\title{
Bizarre stromal cells in Crohn's disease
}

\section{Konstantinos Iliakopoulos ${ }^{\mathrm{a}, \mathrm{d}}$, Menelaos Tzikanoulas ${ }^{\mathrm{b}}$, loannis Papaconstantinou ${ }^{\mathrm{a}}$, Dina Tiniakos ${ }^{\mathrm{b}, \mathrm{c}}$}

Aretaieion Hospital, Medical School, National and Kapodistrian University of Athens, Greece; Newcastle University, UK; Medical School, National and Kapodistrian University of Athens, Greece

Abstract

Lesions with bizarre stromal cells (BSC) have been described in many organs and, although benign, they are frequently misdiagnosed as malignancy. We present the first case of BSC in Crohn's disease with a rare submucosal topography and we review the related literature. We hypothesize that, in Crohn's disease, BSC of myofibroblastic origin may be related to the development of submucosal fibrosis.

Keywords Bizarre stromal cells, gastrointestinal tract, colon, gut, Crohn's disease

Ann Gastroenterol 2019; 32 (2): 1-4

\section{Introduction}

Bizarre stromal cells (BSC) have been reported in many different organs in association with granulation tissue, usually in benign inflammatory polyps or in ulcerated mucosa. BSC have atypical cytological characteristics with diverse shape, vesicular large nuclei with prominent nucleoli and a variable amount of cytoplasm. The differential diagnosis when they are detected in groups includes highgrade carcinoma, sarcoma or melanoma and they also have to be distinguished from cytomegalovirus (CMV)-infected cells or ganglion cells [1].

In the gastrointestinal (GI) tract, BSC have been reported in gastric ulcers, esophageal polyps in patients with reflux esophagitis, granulation tissue near surgical anastomoses, ischemic colitis, pseudopolyps in ulcerative colitis, and anal fibro-epithelial polyps $[2,3]$. In this case report we present an adult man with mildly active Crohn's disease of the colon, modified by treatment, and a focal collection of submucosal

\footnotetext{
a $2^{\text {nd }}$ Department of Surgery (Konstantinos Iliakopoulos, Ioannis Papaconstantinou) and bepartment of Pathology (Menelaos Tzikanoulas, Dina Tiniakos), Aretaieion Hospital, Medical School, National and Kapodistrian University of Athens, Greece; 'Institute of Cellular Medicine, Faculty of Medical Sciences, Newcastle University, Newcastle upon Tyne, NE2 4HH, United Kingdom (Dina Tiniakos); ${ }^{\mathrm{d} D e p a r t m e n t}$ of Anatomy and Surgical Anatomy, Medical School, National and Kapodistrian University of Athens, Greece (Konstantinos Iliakopoulos)
}

Conflict of Interest: None

Correspondence to: Dr Dina Tiniakos, Department of Pathology, Aretaieion Hospital, Vas. Sofias 76, Athens 11528, Greece, e-mail:dtiniak@med.uoa.gr

Received 3 September 2018; accepted 19 November 2018; published online 15 January 2019

DOI: https://doi.org/10.20524/aog.2019.0347
BSC. This is the first reported case of BSC in Crohn's disease and BSC with submucosal topography in the GI tract.

\section{Case report}

A 42-year-old male patient with known treated colonic Crohn's disease presented with terminal ileal stenosis with edema and inflammation of the ileocecal valve. An extensive right colectomy followed by ileostomy was performed. Histological examination showed mild distortion of colonic mucosal architecture with focal erosions and a mixed inflammatory infiltrate with an increased number of eosinophil polymorphonuclear leukocytes, and focal fibrosis and edema of the submucosa, more pronounced in the ileocecal valve. At the upper submucosal layers of the cecum, underneath eroded mucosa, a group of atypical epithelioid, spindle or stellate cells with large, hyperchromatic, polymorphic nuclei and abundant eosinophilic cytoplasm were detected. Some of the cells were multinucleated (Fig. 1). The atypical cells, in the limited number of serial sections where they were still present, were positive by immunohistochemistry for the mesenchymal marker vimentin, the majority were positive for the macrophage marker CD68, while some were positive for $\alpha$-smooth muscle actin (aSMA). They were negative for endothelial markers (CD31, CD34), epithelial markers (pankeratin) (Fig. 2) and CMV. Their immunophenotype was considered indicative of macrophage, fibroblastic or myofibroblastic origin.

\section{Discussion}

In 1982, Isaacson [4] first reported BSC in inflammatory lesions of the esophagus, stomach and rectum. Since then, in addition to the GI tract, BSC have been detected in benign lesions 

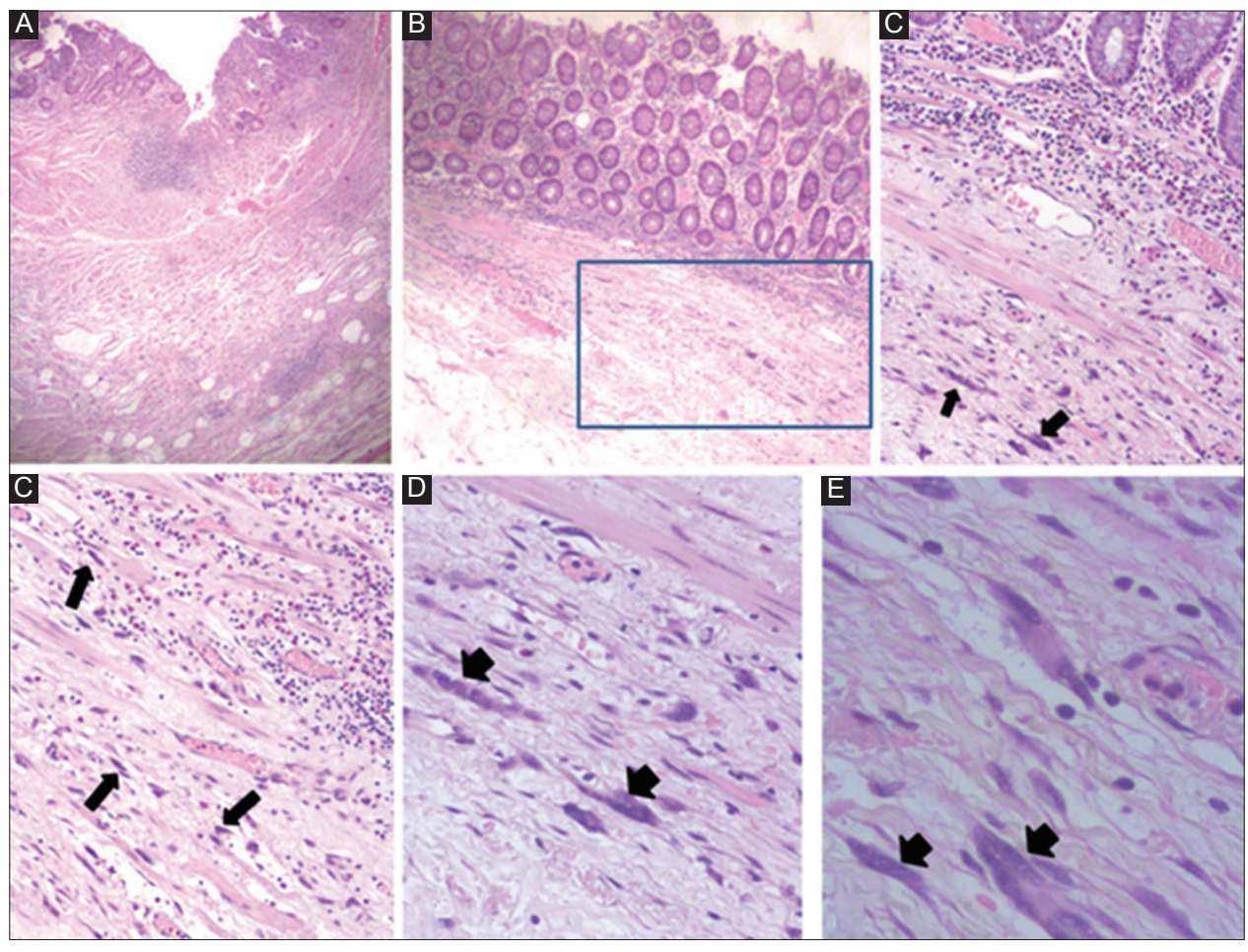

Figure 1 (A) Inflamed mucosa and fibrosed inflamed submucosa in treated Crohn's disease, hematoxylin and eosin $(\mathrm{H} \& \mathrm{E}) \times 20$. (B) Bizarre cells (rectangle) in the upper submucosa, $\mathrm{H} \& \mathrm{E} \times 100$. (C, D) Arrows show the submucosal large spindle-shaped cells in higher magnification in association with a mixed inflammatory infiltrate rich in eosinophils, H\&E $\times 200$. (E, F) Some bizarre cells are multinucleated (arrows in E), while others have large hyperchromatic polymorphic nuclei and abundant eosinophilic cytoplasm (arrows in F), H\&E $\times 400$

of the breast, female reproductive system, prostate, urinary tract, spleen, and paranasal tracts [2]. In the GI tract, BSCs have been encountered in lesions of the gastroesophageal junction, esophagus [5], in relation to gastric ulcers $[2,4,6,7]$, in ischemic colitis [8], and in ulcerative colitis [9,10]. Inflammation appears to be the common underlying condition, regardless of lesion topography [11]. Ours is the first report of BSC in Crohn's disease and we highlight their rare submucosal topography (Table 1).

The first symptoms may include GI bleeding, epigastric pain, abdominal distension, sour regurgitation, belching, heartburn and anemia. A medical history of inflammatory bowel disease (IBD) is not uncommon [5,9]. As mentioned above, lesions containing BSC may occur throughout the GI tract, but the distal part of the esophagus, particularly the region next to the gastroesophageal junction, and the large intestine are most commonly affected [2]. BSC may present within intramural masses or large polyps, rather than within a small polyp or ulcer, which is the typical presentation of pseudomalignant lesions. BSCs have also been identified within ischemic mucosa since the widespread use of endoscopy of GI disorders has increased the frequency with which these lesions are encountered $[4,12,13]$.

On histology, BSC appear as atypical, spindled, stellate, epithelioid or large round cells within inflamed lamina propria or granulation tissue. They have abundant amphophilic or eosinophilic cytoplasm, vesicular nuclei and large eosinophilic nucleoli, which sometimes may be difficult to differentiate from CMV inclusions. Immunohistochemical stains for CMV, however, are always negative [8], as in our case.
BSCs are usually dispersed in a zone under ulcerated or regenerating mucosa but have not been reported in the submucosa to date. BSC most likely represent reactive fibroblasts or myofibroblasts [7,14], an observation strengthened by the fact that they stain strongly for vimentin and sometimes for $\alpha$ SMA. Shekitka et al showed that BSC were positive for vimentin in 20 and aSMA in 7 of 23 cases [2]. In the majority of the studies, Ki-67 immunostaining highlighted their low proliferative rate (labeling index $<1 \%$ ). Mitotic figures are very uncommon and atypical mitoses are absent, while no cytoplasmic mucin or glycogen is detected with special histochemical stains. BSC sometimes lie within inflammatory exudate with associated epithelial elements and may disappear on follow up. Immunostaining for pankeratin is essential to rule out carcinoma.

If the histologic changes are not absolutely convincing, or if the surgical pathologist does not feel confident about establishing a definite benign diagnosis, it is advisable to repeat the biopsy, since it has been reported that atypical regenerative changes can decrease notably or even disappear within 3-5 weeks. A repeat biopsy, clinical follow up and subsequent endoscopic studies are best recommended. Isaacson presented a series of 10 cases in which major resections were performed unnecessarily in three patients following erroneous diagnoses of carcinoma. This is quite worrying, given the fact that a second opinion from a consultant GI pathologist was obtained for the final diagnosis. In the series of Shekitka and Helwig, epithelial or mesenchymal malignancy was incorrectly diagnosed in 6 
Table 1 Case reports of bizarre stromal cells in the gastrointestinal tract

\begin{tabular}{lcccl} 
Author & Year & Ref. & $\mathrm{n}$ & Organ involved/disease \\
\hline Iliakopoulos et al & 2019 & & 1 & Colon \\
Xinqing et al & 2017 & {$[5]$} & 9 & Esophagus \\
Piana et al & 2014 & {$[14]$} & 1 & Esophagus \\
Al-Oqaily et al & 2013 & {$[20]$} & 1 & Esophagus \\
Dhungel \& De Petris & 2013 & {$[11]$} & 2 & Esophagus \\
Wolf et al & 2013 & {$[16]$} & 1 & Esophagus \\
Kamat et al & 2006 & {$[10]$} & 1 & Colon/ulcerative colitis \\
Serra \& Chetty & 2005 & {$[8]$} & 3 & Colon/Ischemic bowel disease \\
Gill et al & 2003 & {$[7]$} & 2 & Esophagus \\
Shekitka \& Helwig & 1991 & {$[2]$} & 33 & $\begin{array}{l}\text { Esophagus }(\mathrm{n}=2) \text {, gastroesophageal junction }(\mathrm{n}=4) \text {, stomach }(\mathrm{n}=5) \text {, large intestine }(\mathrm{n}=21), \\
\text { anal canal }(\mathrm{n}=1)\end{array}$ \\
\hline Isaacson et al & 1982 & {$[4]$} & 7 & Esophagus $(\mathrm{n}=2)$, stomach $(\mathrm{n}=4)$, rectum $(\mathrm{n}=1)$ \\
\hline
\end{tabular}

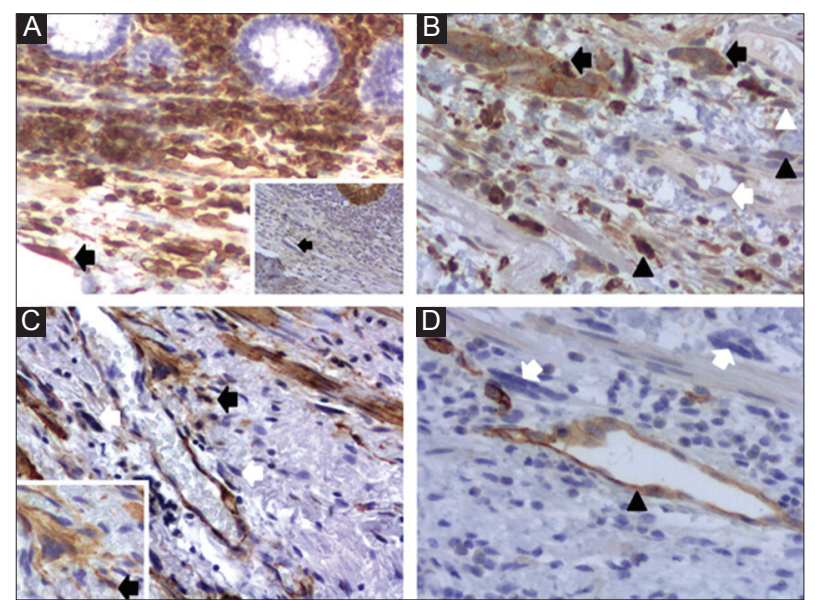

Figure 2 Immunohistochemical characteristics of bizarre submucosal cells (3,3'-diaminobenzidine chromogen): (A) Positivity for vimentin (arrow, $\times 400$ ) and absence of immunoreactivity for pankeratin (inset, arrow, $\times 200$ ) highlights their mesenchymal nature. Lymphocytes and epithelial crypt cells (inset, upper right) act as internal positive control for vimentin and pankeratin, respectively. (B) Multinucleated bizarre cells (black arrows) and some of the mononuclear bizarre cells (black arrowheads) are positive for the macrophage marker CD68, while occasional bizarre cells (white arrowhead) and endothelial cells (white arrow), as expected, are negative $(\times 400)$. (C) Occasional immunopositivity for $a$-smooth muscle actin (aSMA) (black arrow), while most bizarre cells are aSMA-negative (white arrows), $\times 400$. Inset shows an aSMA-positive mononuclear bizarre cell (black arrow) in higher magnification. The adjacent multinucleated cell is negative for aSMA (×600). Smooth muscle cells from the muscularis mucosae (upper right) serve as internal positive control. (D) Submucosal bizarre cells (white arrows) are negative for the endothelial marker CD34, while the endothelial lining of a capillary (black arrowhead) and smooth muscle fibers (upper right) and inflammatory cells serve as internal positive and negative controls, respectively. Immunostaining for cytomegalovirus was negative (not shown)

of $33(18 \%)$ cases with BSC. When 24 of these patients were followed up for an average of 13 months, none of the lesions with BSC progressed to malignancy [2].
In our case of Crohn's colitis, some of the submucosal BSC expressed aSMA in association with vimentin, indicating a possible myofibroblastic origin. Myofibroblasts are found in sites of inflammation in response to chemotactic gradients and are involved in tissue growth and repair, peripheral immune tolerance, inflammation and fibrosis. They produce extracellular matrix (ECM) molecules, resulting in tissue fibrosis as a consequence of chronic inflammation and injury associated with IBD, mainly Crohn's disease [17]. It is possible that, in our case, BSC of myofibroblastic origin along with classical myofibroblasts were responsible for the submucosal fibrosis observed histologically. Newly proposed therapy for Crohn's disease-related fibrosis is now directed toward the immune aspect of this complex chronic inflammatory disorder. New treatments involve disruption of inflammatory signaling pathways that lead to fibrocyte/activated myofibroblast migration, and stem cell therapy to replace these ECM-producing cells and/or enrich the inflammatory microenvironment with less profibrogenic mesenchymal cells [17-19].

In conclusion, BSC in GI lesions may lead to a major diagnostic pitfall. Endoscopic, histological and immunohistochemical characteristics are helpful for an accurate diagnosis. Awareness of the presence of these atypical cells can prevent unnecessary operations, thus having a significant impact on patients' quality of life.

\section{References}

1. Robey SS, Gage WR, Kuhajda FP. Comparison of immunoperoxidase and DNA in situ hybridization techniques in the diagnosis of cytomegalovirus colitis. Am J Clin Pathol 1988;89:666-671.

2. Shekitka KM, Helwig EB. Deceptive bizarre stromal cells in polyps and ulcers of the gastrointestinal tract. Cancer 1991;67:2111-2117.

3. Nakayama M, Wenig BM, Heffner DK. Atypical stromal cells in inflammatory nasal polyps: immunohistochemical and ultrastructural analysis in defining histogenesis. Laryngoscope 1995;105:127-134.

4. Isaacson P. Biopsy appearances easily mistaken for malignancy in 
gastrointestinal endoscopy. Histopathology 1982;6:377-389.

5. Ye X, Li C, Tian Y, Guo C, Yi X. Bizarre stromal cells of the esophagus polyp or papilloma: clinical and pathologic studies of nine cases. Int J Clin Exp Pathol 2017;10:3739-3745.

6. De Petris G, Leung ST. Pseudoneoplasms of the gastrointestinal tract. Arch Pathol Lab Med 2010;134:378-392.

7. Gill P, Piris J, Warren BF. Bizarre stromal cells in the oesophagus. Histopathology 2003;42:88-90.

8. Serra S, Chetty R. Bizarre stromal cells in ischemic bowel disease. Ann Diagn Pathol 2005;9:193-196.

9. Jessurum J, Paplanus SH, Nagle RB, et al. Pseudosarcomatous changes in inflammatory polyps of the colon. Arch Pathol Lab Med 1986;110:833-836.

10. Kamat RN, Amarapurkar AD. Atypical stromal cells in inflammatory pseudopolyps-a case report. Indian J Pathol Microbiol 2006;49:428-430.

11. Dhungel BM, De Petris G. Bizarre stromal cells in the esophagus: report of 2 cases and literature review. Int J Surg Pathol 2013;21:368-372.

12. Chang J, Pathak AS, Dikranian AH, Danial T, Patel HS, Kaswick JA. Benign prostatic stromal hyperplasia with bizarre nuclei. J Urol 2003;170:1951.

13. Yokoyama Y, Yokoyama H, Nagayo T. On biopsy of excavated gastric lesions with special reference to differential diagnosis of atypical regenerative and cancerous epithelia. Stomach Intestine 1974;9:9-20.

14. Piana S, Valli R, Sacchero RI. Bizarre stromal cells in the esophagus: a new case. Int J Surg Pathol 2014;22:383-384.

15. Arista-Nasr J, Rivera I, Martinez-Benitez B, Bornstein-Quevedo L, Orozco H, Lugo-Guevara Y. Atypical regenerative hyperplasia of the esophagus in endoscopic biopsy: a mimicker of squamous esophagic carcinoma. Arch Pathol Lab Med 2005;129:899-904.

16. Wolf EM, Högenauer C, Asslaber M, Langner C. [Inflammatory esophageal polyp with bizarre stromal cells: possible diagnostic pitfall]. Pathologe 2013;34:159-161.

17. Hawkey CJ. Hematopoietic stem cell transplantation in crohn's disease: state-of-the-art treatment. Dig Dis 2017;35:107-114.

18. Forbes GM. Mesenchymal stromal cell therapy in Crohn's disease. Dig Dis 2017;35:115-122.

19. Lo Monaco M, Puzzo L, Brancato F, Torrisi A, Magro G. Endometrial atypical (bizarre) stromal cells: a potential diagnostic pitfall in biopsy. Pathol Res Pract 2004;200:625-627.

20. Al-Oqaily A, Aldauod N, Matalka I. Bizarre stromal cells in the esophagus mimicking invasive malignancy. Proceedings of the $25^{\text {th }}$ Congress of the Arab Division of the International Academy of Pathology 2013:P17D3. [Abstract] 